\title{
Enabling Communication and Navigation Technologies for Future Near Earth Science Missions
}

\author{
David J. Israel ${ }^{1}$, Greg Heckler ${ }^{2}$, Robert J. Menrad ${ }^{3}$ and John J. Hudiburg ${ }^{4}$ \\ National Aeronautics and Space Administration Goddard Spaceflight Center, Greenbelt, MD, 20771 \\ Don M. Boroson ${ }^{5}$, and Bryan S. Robinson ${ }^{6}$ \\ Massachusetts Institute of Technology Lincoln Laboratory, Lexington, MA, 02420 \\ And \\ Donald M. Cornwell ${ }^{7}$ \\ National Aeronautics and Space Administration Headquarters, Washington District of Columbia, 20546
}

\begin{abstract}
In 2015, the Earth Regimes Network Evolution Study (ERNESt) Team proposed a fundamentally new architectural concept, with enabling technologies, that defines an evolutionary pathway out to the 2040 timeframe in which an increasing user community comprised of more diverse space science and exploration missions can be supported. The architectural concept evolves the current instantiations of the Near Earth Network and Space Network through implementation of select technologies resulting in a global communication and navigation network that provides communication and navigation services to a wide range of space users in the Near Earth regime, defined as an Earth-centered sphere with radius of $2 \mathrm{M}$ Km. The enabling technologies include: High Rate Optical Communications, Optical Multiple Access (OMA), Delay Tolerant Networking (DTN), User Initiated Services (UIS), and advanced Position, Navigation, and Timing technology (PNT). This paper describes this new architecture, the key technologies that enable it and their current technology readiness levels. Examples of science missions that could be enabled by the technologies and the projected operational benefits of the architecture concept to missions are also described.
\end{abstract}

\section{Introduction}

$\mathrm{T}$ he Earth Regimes Network Evolution Study (ERNESt) was completed in May 2015. ${ }^{1}$ The study was chartered by NASA's Space Communications and Navigations $(\mathrm{SCaN})$ Program; responsible for all NASA space communication and navigation activities through the Deep Space Network (DSN), Near Earth Network (NEN), and Space Network (SN). The study was comprised of a multi-center team led by NASA's Goddard Spaceflight Center (GSFC). The ERNESt team set out to create a next generation near-Earth space communications and navigation

\footnotetext{
${ }^{1}$ ESC Architect, Exploration \& Space Communications Projects, NASA GSFC/Code 450, Greenbelt, MD 20771, AIAA Senior Member

${ }^{2}$ Electronics Engineer, NASA GSFC/566.0, Greenbelt, MD 20771

${ }^{3}$ Associate Director, Exploration \& Space Communications Projects, NASA GSFC/Code 450, Greenbelt, MD 20771

${ }^{4}$ Mission System Engineer, Exploration \& Space Communications Projects, NASA GSFC/Code 450, AIAA Senior Member

${ }^{5}$ Lincoln Laboratory, Massachusetts Institute of Technology, 244 Wood Street, Lexington, MA 02420

AIAA Member

${ }^{6}$ Assistant Group Leader, Optical Communications Technology, MIT Lincoln Laboratory, brobinson@ll.mit.edu, AIAA Member

${ }^{7}$ Director, Optical Comm Division, Space Comm \& Nav, NASA Headquarters,300 E Street SW, Washington, DC 20024, AIAA Member
} 
architecture for 2025 and beyond with the goal of a wholly-new architecture being in effect by 2040 . This architecture would provide communication and navigation services to missions within $2 \mathrm{M}$ kilometers of the Earth (just beyond the Earth-Sun L2 point); hereafter defined as the "Near Earth Regime". Among its many attributes the ERNESt architecture would also be customizable and scalable to allow room for the inclusion of industry and international partners, helping the network to advance new science and technologies, while driving down commoditized costs.

The resulting ERNESt architectural framework was named the "Space Mobile Network (SMN)". ${ }^{2}$ The SMN seeks to create a spaceflight user community experience analogous to the terrestrial mobile wireless network. Today, a terrestrial mobile wireless user can connect to "the cloud" and have access to others connected to same network at any time. In the same way, SMN-capable users will be able to connect to the SMN architecture, without the humancentered, lengthy prescheduling processes utilized today all along being confident that their data will reach the desired destination.

To illustrate the SMN operations concept, consider a low earth orbiting (LEO) Earth science spacecraft with multiple science instruments. The user spacecraft is continually generating data at steady rate and, in addition, experiences occasional data generation bursts due to science events. A low rate link is continuously available for basic spacecraft commanding and housekeeping telemetry functions. A request is transmitted over this low rate link when the spacecraft determines that it requires services, such as a high data rate link due to the utilization level of its sold state recorder. The user spacecraft's request for service is sent into the SMN using established protocols. After the network determines the next available opportunity to support the mission, the user receives a system response containing service-start and necessary parameters for the event. These could be parameters such as the initial acquisition vector and slew-pattern within the time required for the mission to access the service. When the service time arrives, the user executes the operational procedure for the requested service analogous to stored command processing using the SMN-provided parameters as procedure parameters. The requested service could be provided by a space relay or ground station from any compatible and participating provider (NASA, commercial, international, etc.). For dual-media users the requested service can utilize various radio frequency bands or optical links.

Several key architectural features for the SMN operational concept have been identified including: continuously available low rate forward and return links; high data rate forward and return links; dynamic link and end-to-end path allocation and scheduling; and Position, Navigation, and Time (PNT) services. This paper will build off of these key architectural features with further discussion on Optical Communications, Delay Tolerant Networking (DTN), User Initiated Services (UIS), and PNT. In addition, several use case examples will be discussed to demonstrate the wide reaching user benefits of the SMN.

\section{Optical Communications}

High data rate links direct-to-earth (DTE) or with relay spacecraft may be either RF or optical. Optical spacebased communication links rates ranging from 10's of Mbps to a few Gbps have already been demonstrated for Near Earth crosslinks and for direct-to-Earth downlinks from ranges as far as the Moon. ${ }^{3}$ As technology develops, these highest rate data links are expected to be constantly increasing. The reduction in size, weight, and power required for optical links may also make low rate optical communications links an enabling technology for a lower data rate multiple access system.

DTE communications links from LEO can have higher data rates than LEO-to-GEO relay links due to shorter link distances. A DTE user will typically have a longer wait than a relay system user until an available ground station is in view from the LEO satellite. However, while a typical LEO spacecraft may be in view of a single ground station for as little as $1 \%$ of the orbital period, the link range may be 100 times shorter than a link to a geosynchronous relay. Thus, in principle, the LEO DTE link could support data rates as high as 10,000 times higher than the geosynchronous relay, assuming similar sized terminals, enabling delivery of larger daily data volumes and much smaller terminals in space and on the ground. Optical links are also not constrained by available spectrum. Near-infrared has more than $10 \mathrm{THz}$ of available, unregulated spectrum. As a result, spacecraft manufactures have increased options in the SMN to trade amongst the various variables available to them such as: recorder capacity, packet-level data prioritization, view-period based latency contributions to overall end-to-end latency and mission operations could, with compatible flight systems, switch between DTE and relay service as needed on orbit.

In its study, the ERNESt team did consider that the channel for an optical link to a ground station will vary greatly during a short pass. This path loss will change predictably based on the orbit, but the effects of propagation 
through the atmosphere will be very dynamic. However, for a link that operates at a single rate, analysis has shown that even simple fiber coupling leads to improved performance in terms of total delivered data volume per day. In fact, this improved performance would result in more data delivery capability than a continuous high-rate access to present-day space-based relay systems. ${ }^{4,5}$

Building upon the mission success of LLCD in which data rates from the moon DTE were higher than 600 Mbps, the LCRD Project will demonstrate a GEO optical communications relay capability at over 1 Gbps. ${ }^{6}$ The LCRD architecture demodulates, frame-switches, and remodulates user data, but does not have any onboard channel coding, storage, or network layer processing. Over the course of the two year experiment, the performance of the flight and ground stations hardware and the channel in between will be characterized, as a more complete understanding of the requirements and optimizations for an operational optical relay is developed. In this way, LCRD will transition the technology that has evolved from the Lunar Laser Communication Demonstration to operations.

In order to service many simultaneous users, mostly at LEO, with optical relay service, the ERNESt team determined that it is now practical to build an array of telescopes residing on a GEO satellite. A simple calculation shows that if a symmetrically-sized LEO-to-GEO system could support $1 \mathrm{Gbps}$, then a related symmetrically-sized system with apertures only $32 \%$ as wide could support $10 \mathrm{Mbps}$. With the $10-\mathrm{cm}$ LCRD as a model, then, we see that an array of 3.2-cm telescopes could provide about $10 \mathrm{Mbps}$ full duplex to as many users as there are telescopes in the array. We also note that a GEO telescope needs only steer perhaps +/- 10 degrees to see all LEO satellites. Depending upon how close together the telescopes could be mounted, this means that we could provide $10 \mathrm{Mbps}$ duplex service to 100 simultaneous users with a square array less than about $40-50 \mathrm{~cm}$ on a side.

Each of these telescopes would be fiber coupled to both a transmitter and a receiver, would include a spatial acquisition sensor, and would provide beam stabilization and point-ahead capability. The $1 \mathrm{Gbps}$ aggregated data could then either be switched and routed or be forwarded via a single high-rate link.

Such links could certainly be controlled and configured traditionally, based on pre-arranged knowledge of each user's coming and going. However, we can also add a Demand-Assigned capability for unscheduled usages. Of course, each of the 100 telescopes could be continuously scanning, looking for a new user. A more robust and faster system, though, could be based on a wide field-of-view telescope (viewing the entire globe) with its image mapped onto a focal plane array of detectors, such as a camera. If a pixel were to register a "hit" from a user's uplink transmission, the system would know that he wanted service. With the proper handshake and identity validation, the GEO relay could then hand over control to one of the small telescopes (the uplink beam would also be illuminating it) for $10 \mathrm{Mbps}$ service, or could even hand over control to a high data rate terminal, such as LCRD.

The primary benefit from such an optical multiple access system (OMA) is the potential for a greatly reduced user burden, when compared to an RF-based multiple access system. If continuously available links exist, but too much $\mathrm{SWaP}$, cost, or operational complexity is required to access them, the links will be of limited utility. If there is minimal mission impact to be a multiple access user, these links could begin to be assumed to be part of nearly all missions. This is a critical assumption towards the change in operations concepts, such as User Initiated Services.

\section{Delay Tolerant Networking}

Within the Near Earth region, the significant delays to data delivery are due to availability of links, not the secondslong delays due to the speed of light and communication systems processing. Though the first instinct may be to build out the architecture to maximize availability of high rate links, Near Earth science missions have had end-to-end data delivery latency requirements on the order of hours or days for a large percentage of their data. This science data is already transmitted in a store-and-forward fashion: instrument data is stored onboard, forwarded to a ground station, and then forwarded to the data destination, see Fig. 1. Delay/Disruption Tolerant Network (DTN) protocols can support these store-and-forward operations in a standardized, scalable, and networking based manner. ${ }^{7}$ 
DTN technology development required for near term Near Earth applications are mainly in the hardware required for flight applications and not in software or protocol development. As the onboard data bus and space link data rates increase, flight systems capable of moving and processing data at higher speeds are a requirement, independent of the use of DTN. DTN protocol specific implementations involve possible hardware acceleration of the Bundle Protocol (BP), Licklider Transmission Protocol (LTP), or Bundle Protocol Security (BPSec). Advancements in DTN routing and network management will also be needed as the operational network grows. The implementation of DTN functionality at ground stations, space relay nodes, and user ground systems is also still required.

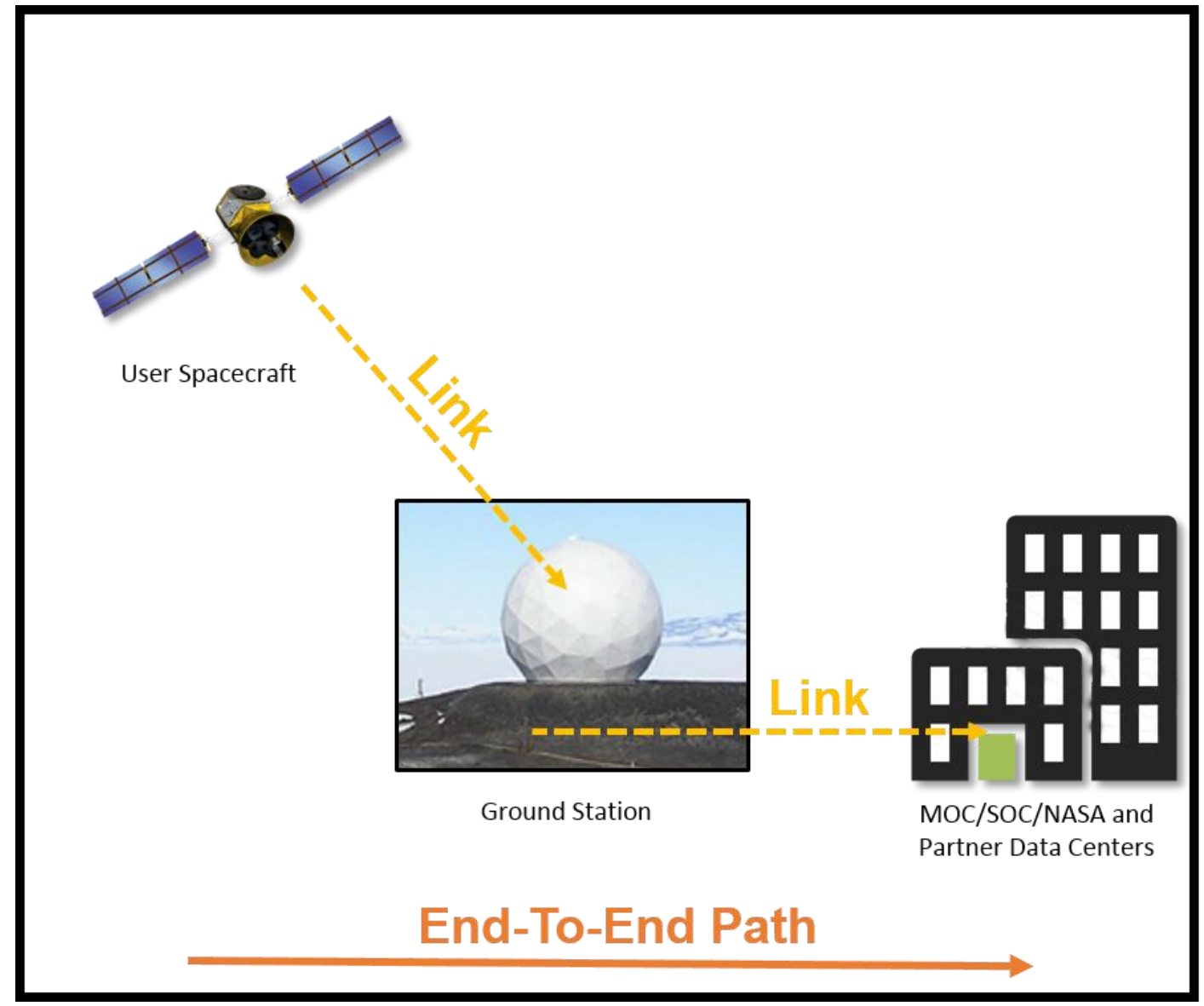

Figure 1. LEO Spacecraft data end-to-end path across multiple link segments

\section{User Initiated Services (UIS)}

From the inception of the space program, mission operations has been overtly scripted by Flight Operations Teams. Whereas recent migration to lights-out operations allow for the execution of that script without human intervention the operational trend has continued to rely upon human intervention to manage the mission timeline being executed. When would contacts occur for data capture? When should station keeping maneuvers be executed? When should upload of command loads or ephemerides occur? These are the questions that humans on the ground address when formulating the execution timeline, which in turn drive the user requests for contact times via the network scheduling process. By contrast, as onboard computing power increases and onboard information such as orbit determination, attitude determination, recorder utilization, etc. then mission designers have the potential to reduce operations costs and complexity through fundamentally different concepts in operations execution. While there has been progress in these areas towards more autonomous operations, the ERNESt team determined that current efforts sought out ways to extract efficiencies from the existing system versus defining a new system in which a new domain for exploiting these concepts my exist. Missions such as a Swift and Fermi/GLAST have employed the low data rate TDRSS Demand Access Service in order to trigger the execution of coordinated campaigns to observe an event through multiple resources as they occur. However, as envisioned by the ERNESt team UIS, offers the user community far greater

$$
4
$$

American Institute of Aeronautics and Astronautics 
autonomy by allowing platform-triggered acquisition of services beyond those low rate services available on demand; and, as a result, provides the means to more fully automate mission execution, address growing numbers of radiating platforms and accommodate greater diversity in those numbers than would be cost-effective through the existing resource approach.

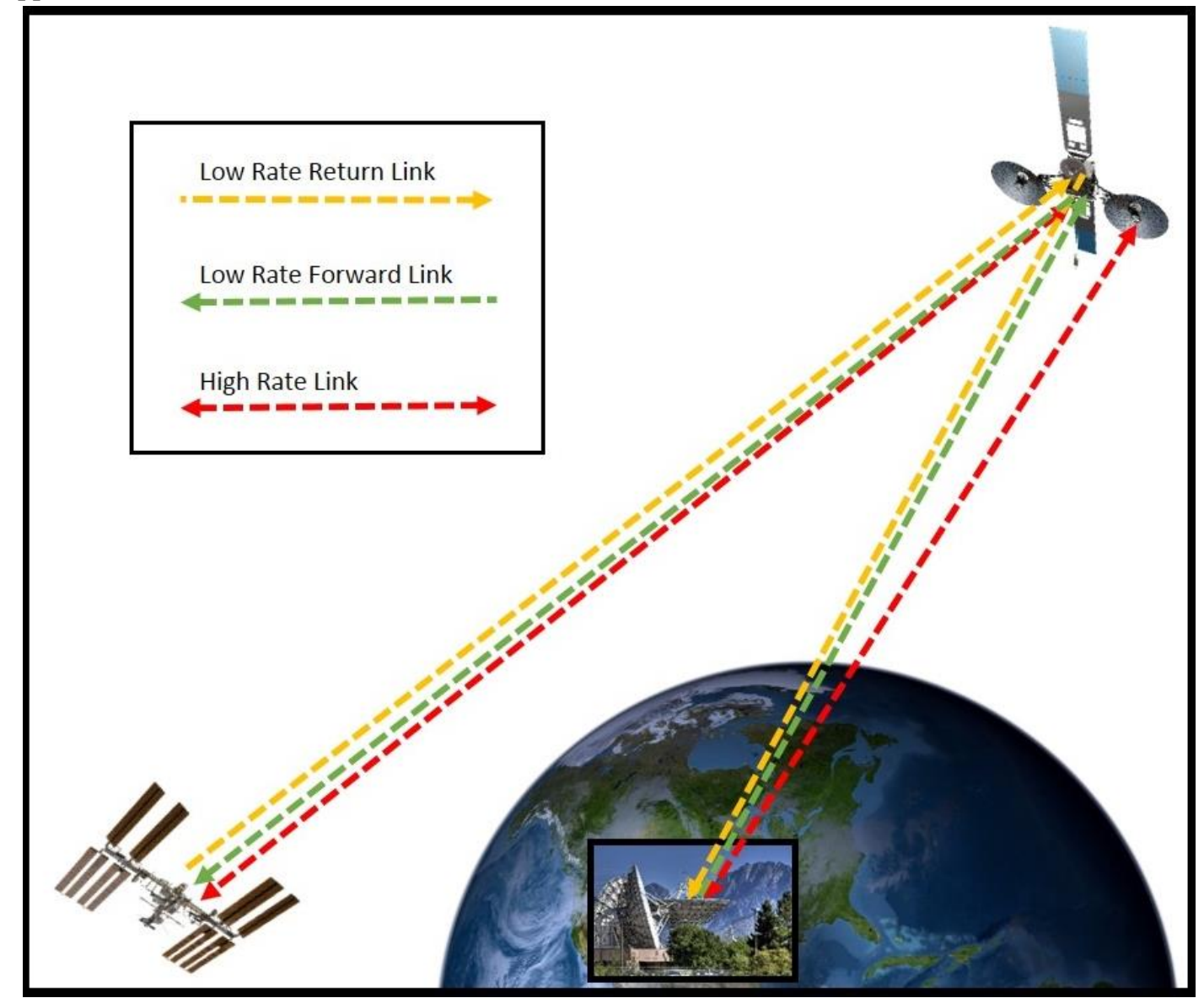

Figure 2. User's may acquire high rate services by requesting them continuously available low rate links

UIS is a method for a network user to access a high rate or other scheduled service on short notice, where "short notice" may be defined an event defined as late as possible while still acquiring the service within the need time. Rather than scheduling services 3-weeks in advance, modifying them 2-weeks in advance and then committing to them 1-week out, a user spacecraft's onboard flight software would have triggers designed into the code that would result in the transmission of a request for service via a UIS-specific protocol over an always-present space link, see Fig. 2. These requests would go back to a central dispatcher function at the core of the SMN architecture where they would be acted upon based upon factors including onboard transmission capabilities (e.g., optical and/or RF). Since service requests can be submitted by any user at any time the SMN would be capable of deconflicting requests using its knowledge of the architecture's nodes and user capabilities. Once the deconflicted assignment has been defined the SMN "dispatcher service" would respond to the service request in one of three ways: acknowledge the service as requested, acknowledge the service request but satisfy it via different yet compatible parameters, or acknowledge the request but decline the support as requested.

The ERNESt team extended the capabilities of any single instance of the SMN architecture through interoperability with partner architectures. Interoperability across various "systems" would increase the number of opportunities for users to have their requests satisfied either as originally requested or via a modified service, see Fig. 3. Specifically, the ability to support users within any given system is determined by the trunk functions used to address the statistically-based needs of the users. Interoperable agreements (i.e., in this context "roaming") affects both system's trunking functions without the corresponding investment of infrastructure. Hence, the coverage maps for UIS compatible users is extended as service providers implement the standards needed to exchange requests thereby 
benefitting all through availability to allow for peering relationships to expand service coverage. Note that the UIS protocol could run over any available link - RF or optical. A cubesat could be receiving service from one ground

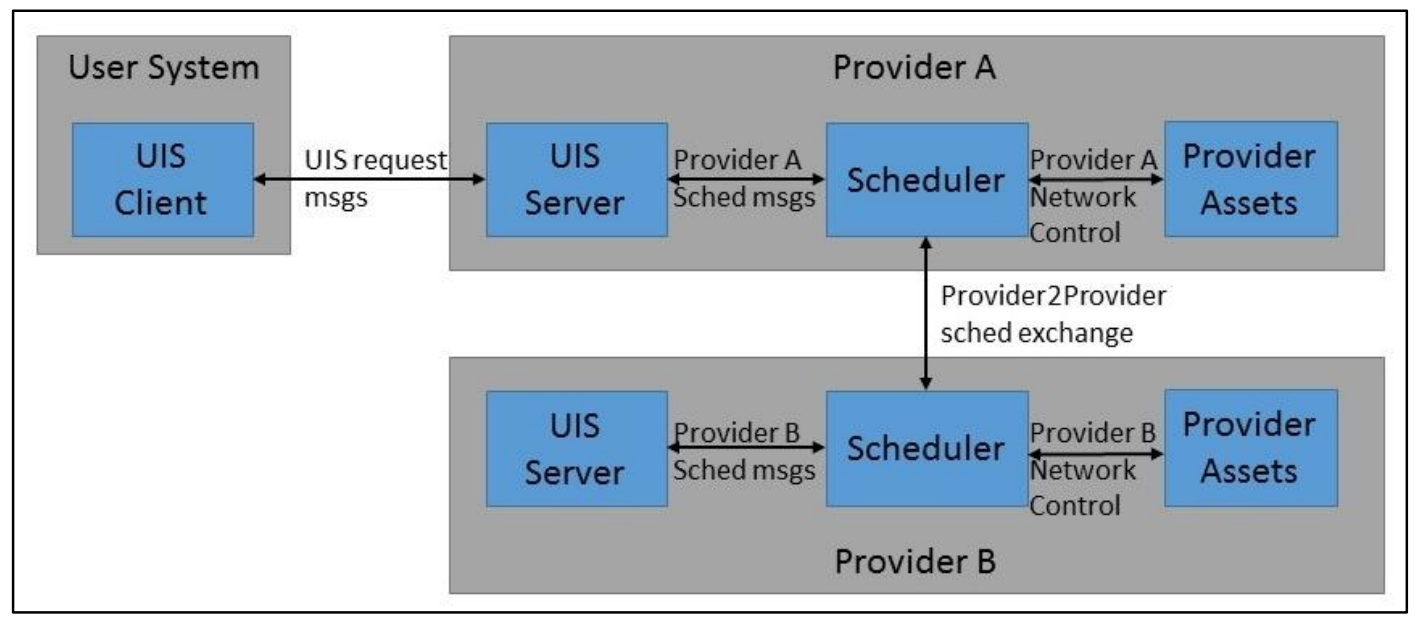

Figure 3. UIS protocols allow for service requests to be fulfilled by multiple providers

station and simultaneously be scheduling its next contact from a different ground station. To realize this interoperable vision both parties would need to insure that both dispatch services were compatible in the means by which service assignments are made, recorded (i.e., avoid contention) and logged (i.e., for mission investigation or billing for services).

The UIS concept requires further development in order to determine the optimum service guarantees. For example, would it be more desirable if the system could guarantee five minutes of contact time within the next thirty minutes or thirty minutes of continuous contact time in the next three hours? More trades involving user operation scenarios is necessary. Other open items include the identification of information required in UIS requests, the definition of the necessary protocols, and how the protocols are carried over the underlying communications layers. Demonstrations of the UIS concept will allow for refinement of the concept, allowing iterations of the implementations of the protocols, as well as the flight and ground implementations.

\section{Position, Navigation, and Timing}

The transition to the SMN envisions greater user autonomy in requesting and accessing communication services, this can be described as a change from a human-machine interface (legacy scheduling) to machine-machine interface (UIS) for request and provisioning of services. The ability to map the physical topology of the network is a necessary to provision service and user state information is a required input to this process. Users currently rely on ground based orbit determination to generate state vectors used by the network. State vectors must be of sufficient accuracy to close the communications link, although generally require less accuracy than science-derived orbit determination requirements. Increased spacecraft autonomy implies a transition from ground based orbit determination processes to onboard processes, else the user will continue to be shackled by long-delay processes that would otherwise undermine the Space Mobile Network concept. ${ }^{8}$

Onboard navigation is a high TRL technology, see Fig. 4. The use of GPS and Global Navigation Satellite System (GNSS) is one mean of delivering onboard navigation. GPS receivers exist of sufficient capability to acquire and track GNSS signals anywhere between LEO and Lunar orbits with hemispherical (low gain) antenna. ${ }^{9}$ These receivers will continue to decrease in size and increase in capability in accordance with Moore's law. The growth in operational GNSS will increase signal availability within while also expanding the region of the GPS and GNSS space service volumes (SSV).

The increase in clock stability over time as normalized on a per dollar, per mass, or per volume basis also facilitates the autonav capability. More stable clocks, such as the chip scale atomic clock, ${ }^{10}$ will increase GNSS receiver accuracy and availability and also a transition from two-way to one-way radiometric techniques. NASA's Deep Space Network (DSN) Deep Space Atomic Clock (DSAC) project is already developing the technology at the root of this architectural change. Ultra-stable oscillators and space rated atomic clocks are TRL9 technologies. 
New types of observations must be incorporated into the menu of sources as they become available. LLCD demonstrated the power of range and range-rate observations (optimetrics) derived from laser communication signals. Although the Lunar Laser Communications Demonstration (LLCD) TRL7 optimetrics demonstration ${ }^{11}$ created a path for technology infusion the Laser Communications Relay Demonstration (LCRD) payload design does not explicitly offer the same capability. GRAIL required $10 \mu \mathrm{m}$ ranging between the two spacecraft, whereas TRL5 optimetrics hardware has shown $2.67 \mu \mathrm{m}$ range and $0.27 \mu \mathrm{m} / \mathrm{s}$ range-rate performance. The orders of magnitude increase in range and range-rate accuracy available on a network with ubiquitous optical communication links is a genuinely new capability yet to be explored by the science community. Note, the techniques demonstrated by LLCD and under current development are derived from the data clock modulated onto the optical carrier; further increases in range and range rate observation performance will be available once direct interrogation of the optical carrier is possible.

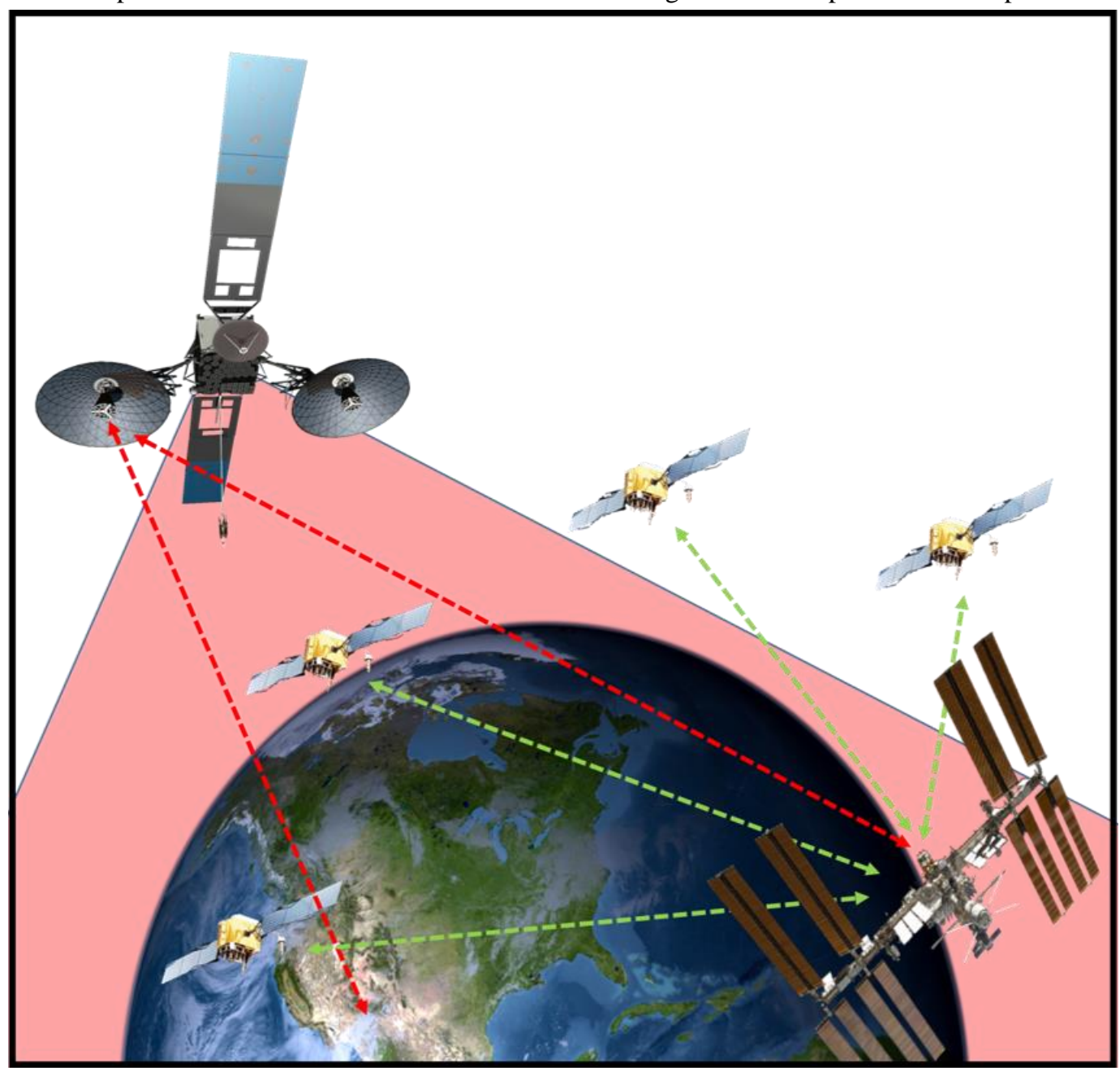

Figure 4. User platform performs autonomous navigation utilizing GPS, GNSS, and space relay signals

Onboard navigation filters are also a TRL9 technology. Optimal sequential filters allow for the user to selfascertain and maintain their orbital state given intermittent observations. Filters exist that process GPS, network radiometrics, and celestial navigation observations simultaneously to generate orbit determination products that support network acquisition requirements and also more stringent science requirements.

Although all the individual technologies exist, and are regularly flown in a piecewise fashion, work remains to integrate the individual technologies into a comprehensive package. By combining the technologies into a single platform a robust autonav capability can be supplied for any user in Near Earth space. Such a platform must be customizable based on the expected orbital regime. A LEO mission might choose to fly a GNSS receiver with a normal $\mathrm{XO}$ or TCXO given the high availability of GNSS signals in this region. A user in a lunar resonant orbit might fly a 
GNSS receiver and an S-band transponder capable of processing one-way forward Doppler observations in order to maintain their onboard filter state during outages. NASA must lead the way in combining these technologies and facilitating their use. NASA's responsibility will shift from technology to standards development as the integrated technology matures and becomes commercially available. Standards that allow individual autonav components to be delivered by multiple vendors while easily integrating into a single onboard system will allow for per-mission customization. CCSDS standards exist for the exchange of navigation information on the ground but will need to be adapted or reworked for use in space.

\section{Space Mobile Network Use Case Examples}

The introduction included a description of an Earth Science mission that was able to use UIS to deliver science data that had been gathered in a non-predicted manner - a science event increased the rate of onboard data generation and the mission was still able to transmit all data in a timely manner. Another example scenario includes collaborative science platforms. In this case, one platform detects an event and transmits a notification to collaborating platforms, while also scheduling up the opportunity to transmit the full data collected. The other platforms receive the notifications, begin their appropriate response (repoint an instrument, increase resolution, etc.), and then transmit their data through the available channels.

Satellite formation flying is a concept of operations already used by NASA missions such as Gravity Recovery and Climate Experiment (GRACE) and Magnetospheric Multiscale (MMS) to meet mission science requirements. Small, micro, and nano satellite buses offer on opportunity to place large numbers of observation platforms into orbit. Currently such small satellite constellations do not actively maintain or modify their orbits. Small satellite maneuvering will be attained as actuator technology scales down to fit within the size, mass, and volume constraints of small satellite buses. Formation flying of small satellites will be achieved through the application of precision orbit determination, maneuver planning, and execution. The SMN concept facilitates small satellite formation flying by providing autonomous orbit determination through the family of PNT technologies discussed above and will offer an on-demand communications channel necessary for maneuver planning and execution.

\section{Conclusions}

When the world recognizes the $100^{\text {th }}$ anniversary of the 1957 launch of Sputnik 1, it is envisioned that the Near Earth regime will be a very active region of travel and exploration. As a result, there will be many existing and new business models. This will result in substantive changes from today in the user community's needs, numbers and diversity, as well as growth in the resources available to mission designers to enable increased complexity of the missions that explore space and exploit the discovered resources.

The ERNESt team determined that extending the script-based utilization of the static infrastructure approach to planning for and then executing mission operations limits all parties to an environment that represents minimal opportunities to extract any new budget efficiencies. In recognition of this the ERNESt team defined a wholly new paradigm for obtaining communications and navigation services during mission operations. The SMN architecture presented herein by the ERNESt team has the architectural and topological features necessary to bridge today's infrastructure to a future state via an accompanying transition strategy that moves spacefaring parties away from the archetype models that has been in place since the inception of spaceflight. Capitalizing upon the proven bandwidth of optical communications a percentage of that bandwidth is now available to carry the realtime channel management data necessary to support on-demand services via the concept of User Initiated Service (UIS). Through the integration of network scheduling to a central virtual "dispatcher" all available nodes are dynamically scheduled Further work is already underway to validate and refine the architecture, to develop the associated technology, and to implement the first demonstrations and early operational capabilities.

The terrestrial mobile network has evolved to $4 \mathrm{G}$ and is on the way to $5 \mathrm{G} .{ }^{12}$ The architecture and ops concept from the user's perspective hasn't really changed over the years, but performance has continued to advance - once it was noteworthy to be able to send a picture from your mobile device and now streaming high definition TV is commonplace. The Space Mobile Network is proposed to be analogous architectural framework for Near Earth space applications. 


\section{ACKNOWLEDGEMENTS}

The authors thank the ERNESt team for their development and recommendation of the Space Mobile Network and Carolyn Crichton, whose editing and graphics support helped to make this paper possible.

NASA's SCaN continues to further define the future space communications and navigation architecture, including Near Earth, Deep Space, and Planetary network elements. The technologies described in this paper are not the full set of candidate technologies to implement the architecture.

\section{References}

1. “Earth Regimes Network Evolution Study,” NASA. 1 May 2015.

2. Israel, David J., Gregory W. Heckler, and Robert J. Menrad. "Space Mobile Network: A Near Earth Communications and Navigation Architecture." IEEE Aerospace Conference 2016, Big Sky, MT, 2016.

3. Israel, David J., Donald M. Cornwell, Gregory D. Menke, W. John Guineau, "Demonstration of Disruption Tolerant Networking across Lunar Optical Communications Links.” AIAA Space 2014, San Diego, CA, 2014.

4. Robinson, B.S., Schieler, C.M., Boroson, D.M. "Large-Volume Data Delivery from Low-Earth Orbit to Ground Using Efficient Single-Mode Optical Receivers”, SPIE LASE. International Society for Optics and Photonics, 2016.

5. Boroson, Don M., et al. "A New Optical Communication Architecture for Delivering Extremely Large Volumes of Data from Space to Ground." AIAA SPACE 2015 Conference and Exposition, 2015.

6. Israel, David J., Bernard L. Edwards, Keith E. Wilson, and John D. Moores. "An Optical Communications Pathfinder for the Next Generation Tracking and Data Relay Satellite." SpaceOps 2014, Pasadena, CA, 2014.

7. Davis, Faith A., Jane K. Marquart, and Greg Menke. "Benefits of delay tolerant networking for earth science missions." In Aerospace Conference, 2012 IEEE, pp. 1-11. IEEE, 2012.

8. Valdez, Jennifer E., Benjamin Ashman, Cheryl Gramling, Gregory W. Heckler, and Russell Carpenter. "Navigation Architecture for a Space Mobile Network." AAS GN\&C Conference, Breckenridge, CO, 2016.

9. Winternitz, Luke, Bill Bamford, Samuel Price, Anne Long, Mitra Farahmand, and Russell Carpenter. "GPS Navigation Above 76,000 km for the MMS Mission." 2016 AAS GN\&C Conference, Breckenridge, CO, 2016.

10. Calero, David, and Enric Fernandez. "Characterization of Chip-Scale Atomic Clock for GNSS navigation solutions." In Navigation World Congress (IAIN), 2015 International Association of Institutes of, pp. 1-8. IEEE, 2015.

11. Stevens, M. L., R. R. Parenti, M. M. Willis, J. A. Greco, F. I. Khatri, B. S. Robinson, and D. M. Boroson. "The lunar laser communication demonstration time-of-flight measurement system: overview, on-orbit performance and ranging analysis." In SPIE LASE, pp. 973908-973908. International Society for Optics and Photonics, 2016.

12. “NGMN 5G White Paper,” NGMN Alliance. 17 February 2015. 